\title{
Des prolongements au modèle de Curzon-Ahlborn du moteur de Carnot
}

\section{Augmented Curzon-Ahlborn modelling of Carnot engine}

\author{
Michel Feidt ${ }^{1}$, Gwilherm Siochan ${ }^{1}$ \\ ${ }^{1}$ Laboratoire LEMTA, Université de Lorraine, CNRS, F-54000 France
}

RÉSUMÉ. Cette publication réexamine le modèle de Curzon-Ahlborn du moteur thermomécanique. Après un rappel historique de modèles gradués (Carnot endo-réversible puis irréversible, Chambadal introduisant l'irréversibilité de transfert à la source) on examine la méthodologie d'optimisation des moteurs thermomécaniques selon le modèle de Curzon et Ahlborn avec un point de vue fondamental, en focalisant sur l'obtention du maximum d'énergie ou de puissance et le rendement associé à ces conditions. II en résulte des résultats nouveaux complémentaires à ceux existants dans la littérature, ainsi qu'une homogénéisation des diverses approches existantes (dont des propositions nouvelles récentes que nous avons faites). Des résultats nouveaux en découlent. On notera plus particulièrement la différence entre les approches en transformations thermodynamiques (selon Curzon et Ahlborn) puis en moyenne de cycle (selon Chambadal) II ressort du présent article l'intérêt confirmé pour la description des irréversibilités en production d'entropie plus qu'en ratio entropique. Cette nouvelle approche peut donner lieu à prolongement actuellement en cours de développement à notre initiative.

ABSTRACT. This paper reconsiders the modelling of Curzon-Ahlborn dedicated to Carnot engine. A modified model is proposed, taking account of the period (duration) of the cycle. It allows a two steps optimization, by following a model incorporating internal irreversibility of the cycle. New results are obtained. We note particularly comparison of various approaches: with thermodynamics transformations according to Curzon and Ahlborn proposal versus mean values over the cycle according to Chambadal proposal. It confirms the interest of the description of irreversibilities with entropy production method preferably to the ratio method. This new approach may give rise to an extension currently under development to our initiative.

MOTS-CLÉS. Moteur de Carnot, Modèle de Chambadal, Modèle de Curzon-Ahlborn amélioré, Action de production d'entropie, Rendement à maximum de puissance.

KEYWORDS. Carnot engine, Chambadal model, improved Curzon-Ahlborn model, Entropy production action, Efficiency at maximum power.

\section{Introduction}

Cette publication considère le cas emblématique de la machine motrice thermomécanique en la complétant de façon très nouvelle et complémentaire par rapport à l'existant.

On propose dans cette introduction une revue sélective des travaux antérieurs. L'objet du présent article est de faire le point sur un sujet relatif aux systèmes et procédés thermomécaniques et plus particulièrement les machines dites thermiques qui sont à l'origine de la révolution industrielle.

Parmi ces machines, on cite :

- les machines de Brayton-Joule, couramment dénommées Turbines à Gaz ou à Combustion [1-7] ;

- les moteurs à combustion interne, dont les moteurs à gazole (ou cycle de Diesel) [8-11] ;

- d'autres moteurs, moins répandus à savoir :

a) les moteurs de Braysson [12] ;

b) les moteurs à air et cycle de Miller standard [13] ;

c) un moteur à trois cycles thermodynamiques [14] ; 
d) les moteurs de Stirling aux nombreuses variantes dont l'intérêt est croissant [15].

On note aussi d'autres systèmes alternatifs très étudiés dont principalement la pile à combustible [16], mais aussi des configurations plus classiques comme les machines à vapeur des centrales thermiques $[17,18]$. Ces deux articles constituent le volet technique et technologique, prolongement pratique actuel des études de Carnot plus fondamentales.

On ajoute aussi des articles traitant des machines à cycles inverses vu leur importance en pratique, mais dont les études restent moins nombreuses dans Int. J. of Thermal Sciences [19].

Aux démarches précédentes il y a lieu d'ajouter des approches plus innovantes ou plus conceptuelles et fondamentales, dont les deux articles proposés par M. Barrère [20, 21]. Nous avons eu la chance d'échanger avec ce dernier auteur, ce qui nous a conforté dans l'intérêt des travaux sur le thème de l'interdépendance des transferts thermiques et de la conversion des énergies dans les systèmes et procédés, dont l'aspect thermomécanique.

Cette thématique a été complétée depuis par:

- des analyses de stabilité [22] ;

- des analyses exergétiques [23];

- des analyses entropiques des irréversibilités [24, 25].

Enfin, les aspects économiques sont aussi une préoccupation de longue date et déjà reliés au respect de l'environnement $[26,21]$.

On insiste pour ce qui nous concerne sur les aspects plus fondamentaux liés à la puissance et aux rendements associés à cette même puissance optimisée. Le défi est de proposer un critère de qualité du moteur plus proche de la réalité fonctionnelle de celui-ci, comme il était proposé dans les références [20, 27-30] en regard de la limite bien connue du rendement de Carnot.

L'article proposé s'appuie sur les modèles précédemment cités (Carnot, Chambadal, puis CurzonAhlborn) pour montrer la progression des connaissances sur l'existence d'optimums successifs d'énergie, puis de puissance et des rendements associés à ces optimums successifs aussi qu'à la production d'entropie ou d'anergie.

Pour ce faire, le plan suivant est adopté. Le paragraphe 2 développera la base historique des modèles de Carnot et Chambadal montrant s'il en était nécessaire que ces derniers modèles ont des racines plus profondes dans l'histoire de la Thermodynamique. Le paragraphe 3 abordera l'optimisation des moteurs thermomécaniques selon le modèle de Carnot, puis Chambadal, en insistant sur la méthodologie des études :

- différents choix pour la fonction objectif ;

- différents choix pour les contraintes ;

- études de sensibilité paramétrique : distinction entre variable et paramètre, dont la connexion aux conditions aux limites ne peut pas être ignorée. D'importantes conséquences en résultent, dont certaines seront rapportées.

On rapellera quelques résultats, pour illustrer la démarche progressive (graduée) utilisée, conduisant à une optimisation séquentielle dont l'aboutissement fournit l'optimum-optimorum recherché, correspondant (dans le cas général irréversible) au rendement de Carnot qui constitue la limite réversible inatteignable. 
Le paragraphe 4 sera dedié aux optimisation séquentielles en énergie du moteur endoreversible et adiabatique de Curzon-Ahlborn : 4.1 rappel de l'optimisation en energie selon ces auteurs. 4.2 premiére proposition d'optimisation par rapport aux variables $G_{i} .4 .3$ optimisation en puissance motrice par rapport aux variables $t_{i}$ en temps fini soumis à la contrainte de finitude (4).

Le paragraphe 5 ajoute une optimisation séquentielle en énergie et puissance du moteur de CurzonAhlborn modifié dans le cas endo-réversible.

Le paragraphe 6 consistera en une discussion-synthèse de résultats unifiant d'assez nombreux travaux de la littérature dont ceux publiés dans R.G.T. devenu I.J.T.S.

Des prolongements et perspectives existantes ou à venir seront enfin exposés.

\section{Base historique des modèles de moteur de Carnot et de Chambadal}

Les machines thermomécaniques (les moteurs thermiques) sont à l'origine de la révolution industrielle. Elles sont aussi à l'origine de la naissance d'une science, la Thermodynamique phénoménologique, avec des aspects applicatifs, mais aussi fondamentaux. Le moteur de Carnot apparait de ce fait comme le cas d'école de référence, mais dans sa version initiale, il est limité à une approche selon la Thermodynamique de l'Equilibre (T.E). Par ailleurs, les études initiales ont été focalisées sur le rendement au sens du premier principe, $\eta_{\mathrm{I}}$, extension de la notion antérieure de rendement mécanique. Il est bien connu que le rendement dit de Carnot ne dépend que des deux températures des thermostats, $T_{H S}$ (chaud) et $T_{C S}$ (froid). Cette dernière température se confond souvent avec la température ambiante ou de l'environnement, $T_{0}$ (elle aussi supposée constante).

Sur un cycle, le moteur thermomécanique convertit une partie de l'énergie calorifique apportée par la source, $Q_{H S}$, en énergie mécanique, $W$ (voir Figure 1). Mais il rejette au puits de chaleur une énergie thermique non convertie $Q_{C S}$ [31]. Il en résulte la relation bien connue :

$$
\eta_{\mathrm{I}}=\frac{W}{Q_{H S}}=1-\frac{T_{C S}}{T_{H S}}
$$

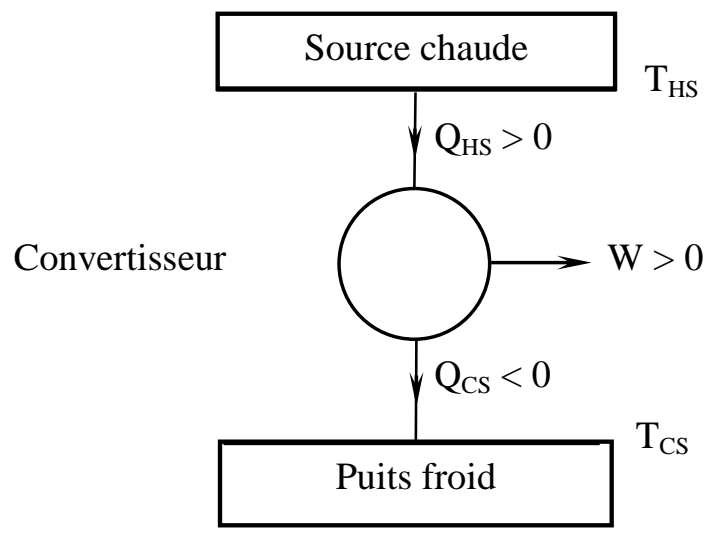

Figure 1. Machine thermique ditherme et convention de signe associée

Par contre dans les mêmes conditions de réversibilité, qui impose la condition de staticité, la période du cycle $\tau$ doit tendre vers l'infini, et par voie de conséquence, il en résulte une puissance moyenne de cycle $\overline{\dot{W}}$ nulle conformément à :

$$
\overline{\dot{W}}=\frac{W}{\tau}
$$


Ainsi depuis les années 1980 environ s'est développée une Thermodynamique en Temps Fini (TTF ou FTT en anglais), principalement endoreversible [32,33]. Nous avons découvert depuis que ces travaux ont été précédés de façon partielle bien avant par Chambadal et Novikov [34, 35], pour Chambadal sur une configuration supposant un régime dynamique stationnaire, pour Novikov en raisonnant sur les énergies du cycle. Dans les deux approches, la finalité reste une efficacité (rendement) associé au maximum de puissance ou d'énergie.

D'autres travaux ont poursuivi le même but, bien avant Chambadal et Novikov, comme le montre les références [36,37] et font même apparaître en plus du rendement à maximum de puissance (appelé coefficient économique [38, page 851]), le facteur de qualité ou rendement au sens du second principe [38, page 851] et [31, p.149]. On notera que dans la 2ème référence, ce rendement était qualifié d'efficacité, comme rendant compte des écarts à la réversibilité.

On retiendra pour l'heure qu'il reste à approfondir l'influence des irréversibilités, la démarche la plus courante considérant les irréversibilités associées aux transferts thermiques (machines endoréversibles) et moins fréquemment les irréversibilités internes au convertisseur.

La littérature révèle que les deux démarches principales pour une caractérisation systémique des irréversibilités de conversion sont :

- la méthode du ratio entropique paraissant sous des formes différentes dans l'article de Novikov [35], puis Ibrahim et al [39]. Il semble utile ici de clarifier les positions, comme il sera proposé au paragraphe 3 ;

- la méthode du forfait de production d'entropie $\Delta S_{I}$ pour un cycle. C'est ce que nous préconisons depuis de nombreuses années [40] et qui sera aussi développer au paragraphe 3.

La complémentarité des deux méthodes précédentes sera illustrée sur le cas simple du moteur de Carnot, en limitant l'étude au modèle de Carnot exoréversible (ou endoirréversible).

D'assez nombreuses études [41, partie 2] rapportent alors de l'incidence de :

- la forme des lois de transfert ;

- de la nature des sources et puits (thermostats ; écoulement de fluides sans changement d'état) ;

- du choix de la fonction objectif ;

- de la considération de contraintes complémentaires ;

- de la présence ou non (adiabaticité) de pertes thermiques ;

- de la description des irréversibilités (méthode globale conduisant à identification ; méthode mécaniste, qu'on pourrait qualifier de méthode des pertes séparées [41, partie1].

On propose ici une approche radicalement nouvelle s'appuyant sur l'entropie de transfert $\Delta S$ (dont la référence entropique sera précisée ) qui est une condition nécessaire d'existence du cycle [42, 43]. A cette entropie de transfert sont associées les irréversibilités de transfert thermique, auxquelles s'ajoute la production globale d'entropie du convertisseur $\Delta S_{I}$, dont on montrera la relation avec le ratio entropique introduit par Novikov.

Pour des raisons de concisions, l'illustration principale de cette approche en Thermodynamique du Non-Equilibre (TNE) ne sera réalisée que par la détermination de l'optimum d'énergie, puis de puissance du moteur, en même temps que les rendements associés et la production totale d'entropie du système. Des conséquences importantes en résulterons.

En partant de l'article de Curzon et Ahlborn [32] qui est à l'origine du « nice radical » : 


$$
\eta_{I}(M A X W)=1-\sqrt{\frac{T_{C S}}{T_{H S}}}
$$

Le rendement au maximum d'énergie du moteur de Carnot endoréversible ne dépend que de la température du thermostat chaud $T_{H S}$, et de celle du thermostat froid $T_{C S}$, en l'absence de pertes thermiques (système adiabatique).

Modéliser l'évolution du rendement au maximum d'énergie du moteur de Carnot endoréversible en fonction du rapport $\frac{T_{H S}}{T_{C S}}$ permet de voir qu'il n'est pas réaliste d'espérer obtenir un rendement proche de 1.

Après cette première optimisation énergétique aboutissant à (3), les auteurs ajoutent une deuxième optimisation, en puissance moyenne de cycle $\bar{W}$, par rapport aux variables durées des transformations tout particulièrement isothermes $t_{H}, t_{C}$. Les auteurs sutilisent alors l'hypothèse que les durées de transferts mécaniques le long des isentropiques sont très inférieures aux durées des transferts thermiques, mais que ces durées sont néanmoins proportionnelles; d'où la durée totale ou période du cycle :

$$
\tau=\alpha\left(t_{H}+t_{C}\right)=\alpha \tau^{\prime}
$$

avec $\alpha=1+\varepsilon, \varepsilon$ petit.

L'optimisation portant cette fois sur les quatre variables $T_{H}, T_{C}, t_{h}, t_{C}$, le maximum de puissance moyenne devient :

$$
\operatorname{MAX}(\overline{\dot{W}})=\frac{K_{H}^{\prime} K_{C}^{\prime}}{\alpha} \frac{\left(\sqrt{T_{H S}}-\sqrt{T_{C S}}\right)^{2}}{\left(\sqrt{K_{H}^{\prime}}+\sqrt{K_{C}^{\prime}}\right)^{2}}
$$

Il apparaît donc dans le cas adiabatique endo-réversible une nouvelle optimisation possible, en remarquant que les conductances de transfert thermique des transformations isothermes doivent être finies, limitées par la contrainte de finitude suivante :

$$
K_{H}^{\prime}+K_{C}^{\prime}=K_{T}^{\prime}
$$

Ceci constitue le point de départ des prolongements au modèle de Curzon-Ahlborn, objet du présent article.

\section{Optimisations des moteurs thermomécaniques}

\subsection{Moteur de Carnot exo-réversible (endo-irréversible)}

\subsubsection{Description et analyse du cycle irréversible}

Le moteur est considéré en contact avec deux thermostats de température $T_{H S}, T_{C S}$ (Figure 2) et sa modélisation reprend en le complétant un travail au cours de publication [44]. On note que l'extension en réservoirs finis sera faite par la suite.

Le cycle irréversible est connu à partir des points d'état 1 (début de l'isotherme haute température), 2 (sortie de l'isotherme haute température et début de la détente adiabatique), 3 (fin de la détente adiabatique et début de l'isotherme basse température), 4 (sortie de l'isotherme basse température et début de la compression adiabatique jusqu'au point 1).

On note ici que les commutations entre isothermes et adiabatiques sont supposées instantanées (ou rappelle qu'une adiabatique diffère d'une isentropique par la présence d'irréversibilités). De même, on 
observe que si les deux isothermes réelles sont bien identifiées sur le diagramme, il n'en est pas de même pour les adiabatiques reliant 2 à 3 , puis 4 à 1 . En effet si toute l'irréversibilité entre 2 et 3 a lieu sur l'isotherme $T_{H S}$ (par exemple à la commutation), la transformation suivra le chemin 2-3'-3. Dans ce cas toute l'irréversibilité est reportée vers l'isotherme haute et la détente 3'3 est isentropique.

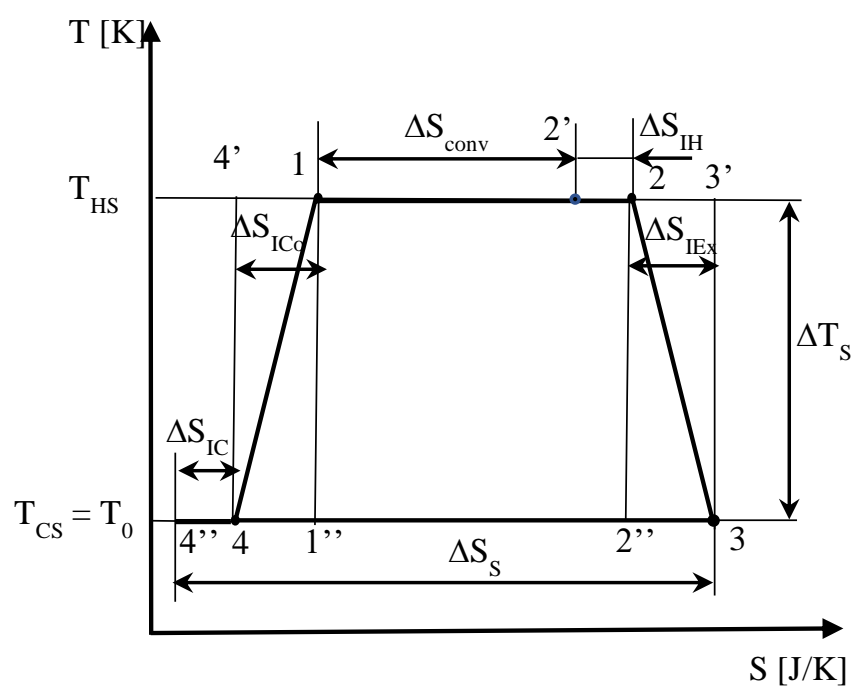

Figure 2. Cycle de Carnot endo-irréversible : les diverses entropies produites et de transfert

A l'inverse, si toute l'irréversibilité se produit sur l'adiabatique juste avant la commutation sur l'isotherme basse température, le chemin suivi sera sur la limite interne 2-2"'-3. Toutes les courbes inscrites dans le rectangle 2-2' '-3-3'-2 satisfaisant au critère d'entropie croissante de l'adiabatique sont possibles. Le fait de tracer une transformation linéaire dans le diagramme $T$-S entre 2 et 3 constitue une solution médiane plausible.

Le même discours peut être renouvelé pour la compression adiabatique entre 4 et 1 . En bref toutes les courbes inscrites dans le rectangle 4-4'-1-1' '-4 et satisfaisant au critère d'entropie croissante de l'adiabatique sont possibles. Ceci complète donc la description particulière de Wang.

\subsubsection{Le modèle analytique en énergie sans pertes thermiques}

Il résulte de la Figure 2 un bilan entropique tel que :

$$
\Delta S_{S}=\Delta S_{\text {conv }}+\Delta S_{I}
$$

avec $\Delta S_{S}=\frac{Q_{C}}{T_{C S}}$, entropie de transfert thermique du convertisseur vers le puits froid,

$\Delta S_{c o n v}$, entropie de transfert thermique utile ou mécanisée dans le convertisseur,

$\Delta S_{I}, \quad$ production totale d'entropie du cycle avec successivement :

$$
\Delta S_{I}=\Delta S_{I H}+\Delta S_{I E x}+\Delta S_{I C}+\Delta S_{I C o}
$$

où $\Delta S_{I H}$, production d'entropie sur l'isotherme haute température,

$\Delta S_{I E x}$, production d'entropie sur la détente adiabatique,

$\Delta S_{I C}$, production d'entropie sur l'isotherme basse température,

$\Delta S_{I C o}$, production d'entropie sur la compression adiabatique.

On remarquera ici que $\Delta S_{\text {conv }}<\Delta S_{H}$ vu que le bilan entropique coté source s'écrit : 


$$
\Delta S_{H}=\Delta S_{c o n v}+\Delta S_{I H}=\frac{Q_{H}}{T_{H S}}
$$

Le fait de négliger les pertes thermiques entre source et puits permet de simplifier l'approche pour analyser plus finement les aspects liés à l'irréversibilité interne au cycle. Le lecteur intéressé pourra se reporter à un article en cours de publication [44].

Le bilan d'énergie du convertisseur fournit l'expression de l'énergie mécanique produite :

$$
\begin{aligned}
& W=Q_{\text {conv }}-Q_{C}=T_{H S} \Delta S_{c o n v}-T_{C S} \Delta S_{S} \\
& W=\left(T_{H S}-T_{C S}\right) \Delta S_{c o n v}-T_{C S} \Delta S_{I}
\end{aligned}
$$

où

$$
W=\left(T_{H S}-T_{C S}\right) \Delta S_{S}-T_{H S} \Delta S_{I}
$$

Les expressions (11) et (12) fournissent respectivement l'énergie mécanique disponible dans le cas où la limitation provient des conditions aux limites à la source haute température (approche séquentielle des transformations en partant de la source), par opposition au cas où la limitation provient des conditions aux limites au puits (approche simultanée : toutes les transformations sont considérées au même instant pour anticiper la fermeture du cycle).

Lorsque la limitation est à la source, on retrouve le théorème de Gouy-Stodola, si $T_{C S}$ se confond avec la température ambiante $T_{0}$. Le maximum d'énergie vaut :

$$
\operatorname{Max}_{H}=\Delta S_{H S} \cdot \Delta T_{S}
$$

avec $\Delta S_{H S}=\Delta S_{H}$ dû à l'adiabaticité et

$$
\Delta T_{S}=T_{H S}-T_{C S}
$$

Lorsque la limitation est au puits de chaleur, $T_{H S}$ est facteur de la production d'entropie, et le maximum d'énergie devient :

$$
\operatorname{Max}_{C}=\Delta S_{C S} \Delta T_{S}=\Delta S_{S} \Delta T_{S}
$$

Il est intéressant de donner les expressions des rendements au sens du premier principe dans ces deux cas. On trouve respectivement à partir de (11) :

$$
\eta_{\mathrm{IH}}=\eta_{C}\left(1-\frac{\Delta S_{I H}}{\Delta S_{H S}}\right)-\frac{T_{C S}}{T_{H S}} \cdot \frac{\Delta S_{I}}{\Delta S_{H S}}
$$

Puis à partir de (12)

$$
\eta_{\mathrm{IC}}=\eta_{C} \frac{\Delta S_{S}}{\Delta S_{S}-\Delta S_{I}+\Delta S_{I H}}-\frac{\Delta S_{I}}{\Delta S_{S}-\Delta S_{I}+\Delta S_{I H}}=\frac{\eta_{C} \cdot \Delta S_{S}-\Delta S_{I}}{\Delta S_{S}-\Delta S_{I}+\Delta S_{I H}}
$$

Les expressions ci-dessus de $\operatorname{Max}_{i}$ et $\eta_{\mathrm{I} i}$ montrent l'importance des références et des couplages, comme il sera précisé dans la suite de l'article. Cette démarche est nouvelle à notre connaissance et mérite d'être considérée attentivement, vu l'influence fondamentale sur les résultats.

Nous développons ci-après l'étude en choisissant pour entropie de transfert de référence $\Delta S_{\text {conv }}=\Delta S$, puisque nous caractérisons le moteur de Carnot endo-irréversible. En factorisant $\Delta S_{\text {conv }}$ dans l'expression (11), il vient :

$$
W=\Delta S\left[\left(T_{H S}-T_{C S}\right)-T_{C S} \frac{\Delta S_{I}}{\Delta S}\right]
$$


On voit apparaitre alors le rapport $\Delta S_{I} / \Delta S$ correspondant au degré d'irréversibilité $A$, introduit par Novikov tel que [35]:

$$
\eta_{I}=1-\frac{T_{C S}}{T_{H S}}\left(1+\frac{\Delta S_{I}}{\Delta S}\right)
$$

Toutefois la référence de Novikov est celle de la chaleur disponible à la source $\left(\Delta S_{H S}\right.$ et non $\left.\Delta S\right)$; donc $A=\frac{\Delta S_{I}}{\Delta S_{H S}}$.

Pour plus de clarté, nous appellerons $d_{I}$ le degré d'irréversibilité introduit dans (19) :

$$
d_{I}=\frac{\Delta S_{I}}{\Delta S}
$$

Ce paramètre est à relier aux différents facteurs d'irréversibilité considérés par Ibrahim et al [39]. On considère ici uniquement le ratio entropique, souvent noté par $I$ et défini par le bilan entropique sur le cycle irréversible selon ces auteurs il vient :

$$
\frac{Q_{H}}{T_{H S}}-I_{C} \frac{Q_{C}}{T_{C S}}=0
$$

L’expression (22) du ratio entropique qui apparaît est celui associé au puits :

$$
I_{C}=\frac{\Delta S_{12}}{\Delta S_{43}}
$$

Conformément aux principes thermodynamique, $I_{C}$ est inférieur à l'unité. Mais il est aussi possible de choisir $I_{H}$, en remarquant que :

$$
I_{H}=\frac{\Delta S_{43}}{\Delta S_{12}}
$$

Le ratio entropique $I_{H}$ paraît préférable, car c'est un coefficient d'amplification entropique à la traversée du convertisseur (production d'entropie). On précise que pour le moteur de Carnot exoréversible $I_{H}=I_{H S}=I$. De plus, on trouve aisément que :

$$
I=1+d_{I}
$$

à la condition de choisir comme entropie de référence $\Delta S$.

D'où il vient à partir de (19) et (20), respectivement :

$$
\begin{aligned}
& W=\Delta S\left[\left(T_{H S}-T_{C S}\right)-T_{C S}(I-1)\right]=\Delta S\left[\left(T_{H S}-I \cdot T_{C S}\right)\right] \\
& \eta_{I_{c o n v}}=1-I \frac{T_{C S}}{T_{H S}}
\end{aligned}
$$

L'irréversibilité interne diminue le travail et le rendement qui est ici un rendement de conversion. Il reste à analyser comment $I$ (méthode de ratio), ou $\Delta S_{I}$ (méthode directe ou du forfait) varient avec les grandeurs du cycle.

On peut supposer raisonnablement que $\Delta S_{I}$ et (ou) $I$ dépendent de $\Delta S$ et (ou) de $\Delta T_{S}$. Il vient alors deux fonctions dont la forme générale inconnue à ce jour s'écrit :

$$
\begin{aligned}
& \Delta S_{I}=f_{S I}\left(\Delta T_{S}, \Delta S\right) \\
& I=f_{I}\left(\Delta T_{S}, \Delta S\right)
\end{aligned}
$$


S'il existe un optimum du travail mécanique $W$, il doit alors satisfaire la condition nécessaire donnée par l'un des couples d'équations suivantes en supposant que les deux variables sont indépendantes :

$$
\begin{gathered}
\left\{\begin{array}{c}
\frac{\partial W}{\partial \Delta T_{S}}=\Delta S-T_{C S} \frac{\partial f_{S I}}{\partial \Delta T_{S}}=0 \\
\frac{\partial W}{\partial \Delta S}=\Delta T_{S}-T_{C S} \frac{\partial f_{S I}}{\partial \Delta S}=0
\end{array}\right. \\
\left\{\begin{array}{c}
\frac{\partial W}{\partial \Delta T_{S}}=\Delta S\left[1-T_{C S} \frac{\partial f_{I}}{\partial \Delta T_{S}}\right]=0 \\
\frac{\partial W}{\partial \Delta S}=\Delta T_{S}-T_{C S} \frac{\partial f_{I}}{\partial \Delta S}=0
\end{array}\right.
\end{gathered}
$$

On remarque enfin que l'existence du cycle dépend des deux variables, $\Delta S$ extensive, $\Delta T_{S}$ intensive, qui doivent être strictement positive avec nos conventions. Ce développement pourra donner lieu à des prolongements ultérieurs selon l'évolution de la connaissance concernant la production d'entropie.

\subsection{Le moteur selon Chambadal}

L'ensemble de la méthodologie exposée au paragraphe précèdent va être illustrée sur l'exemple du moteur de Chambadal. La représentation de la Figure 3 montre qu'en plus de l'irréversibilité interne $\Delta S_{I}$, il apparait l'irréversibilité de transfert thermique au bout chaud.

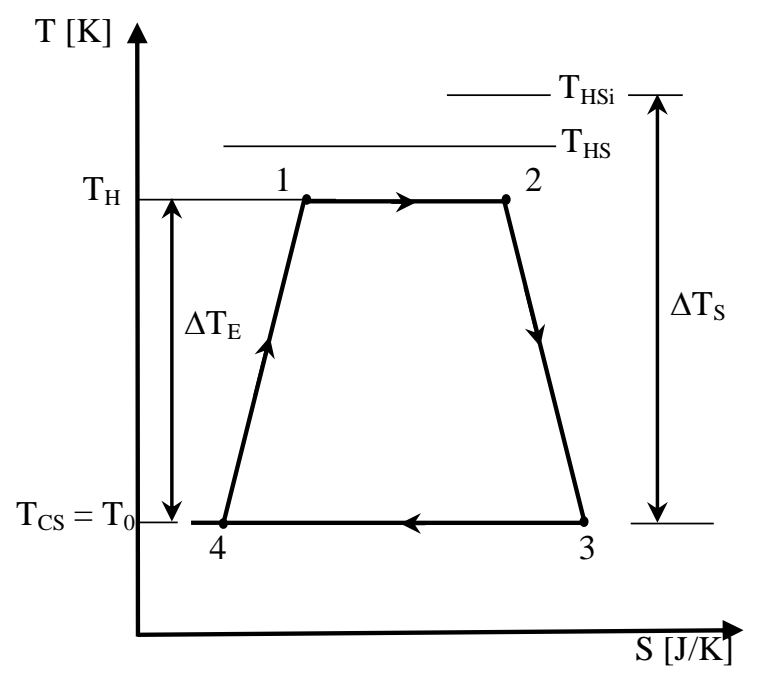

Figure 3. Diagramme T-S d'un cycle irréversible associé au moteur de Chambadal

Le système thermo-mécanique de Chambadal fait intervenir une source de chaleur sensible de débit calorifique fini :

$$
\dot{C}_{H}=\dot{m} c_{P H}
$$

avec $m_{H}$, débit massique fini du fluide source ;

$c_{P H}$, chaleur spécifique massique à pression constante du fluide source (supposée constante).

La température d'entrée du fluide source est $T_{H S i}$. La température de sortie de ce même fluide doit être supérieure à $T_{H}$. Avec l'hypothèse d'un régime dynamique stationnaire et usage de la méthode de l'efficacité et des nombres d'unités de transfert pour les transferts thermiques au bout chaud [40,42], il vient pour le flux transféré au fluide cyclé :

$$
\dot{Q}_{H}=\varepsilon_{H} \dot{C}_{H}\left(T_{H S i}-T_{H}\right)
$$


Par souci de simplification on définit ci-après un moteur de Chambadal en contact avec un thermostat à $T_{H S}$ (moteur de Chambadal adapté), tel que le flux de transfert thermique au bout chaud devient :

$$
\dot{Q}_{H}=k_{H} A_{H}\left(T_{H S}-T_{H}\right)=K_{H}\left(T_{H S}-T_{H}\right)
$$

avec $K_{H}$, conductance de transfert thermique entre le thermostat et le fluide cyclé.

On notera que les deux expressions de $\dot{Q}_{H}$ permettent moyennant une durée $\Delta t$, de remonter à l'énergie échangée. On peut donc écrire de façon générale [42,43] :

$$
Q_{H}=G_{H}\left(T_{H S i}-T_{H}\right)=T_{H} \cdot \Delta S_{H}
$$

avec $G_{H}$, dimension physique finie au bout chaud.

Remarque : pour un moteur sans pertes thermiques, on précise que : $Q_{H}=Q_{H S}=T_{H S} \cdot \Delta S_{H S}$.

\subsubsection{Optimisation du moteur de Chambadal adapté sans la contrainte d'entropie de transfert}

L'optimisation par la méthode du ratio entropique est conforme à l'approche proposée par Ibrahim.

A l'équation (34), il y a lieu d'ajouter l'équivalent de l'équation (22), le bilan entropique sur le fluide cyclé :

$$
I_{H} \frac{Q_{H}}{T_{H}}=\frac{Q_{C}}{T_{0}}
$$

Le calcul ne sera pas prolongé ici pour des raisons de concision. On se contentera d'aborder l'optimisation par le modèle en production d'entropie $\Delta S_{I}$.

Le bilan entropique prend une forme analogue à celle de la relation (33).

\subsubsection{Optimisation du moteur de Chambadal adapté avec la contrainte d'entropie de transfert}

On a vu sur l'expression (34) que le couplage entre la source et le convertisseur induisait une interdépendance de $T_{H}, G_{H}$ et $\Delta S_{H}$ exprimée par :

$$
T_{H}=\frac{G_{H}}{G_{H}+\Delta S_{H}} T_{H S i}
$$

En choisissant $\Delta S=\Delta S_{H}$, entropie de transfert de référence en entrée du moteur avant conversion, on ne tient compte que du transfert d'entropie source-convertisseur. On en déduit aisément l'expression de $W$ en fonction de la référence $\Delta S$ :

$$
W(\Delta S)=\left(\Delta S-\Delta S_{I H}\right)\left(\frac{G_{H} T_{H S i}}{G_{H}+\Delta S}-T_{0}\right)-T_{0} \Delta S_{I}
$$

On voit que $W$ sera maximum par dérivation de (37), mais en tenant compte de la dépendance non seulement de $\Delta S_{I}$ mais aussi de $\Delta S_{I H}$ en fonction de $\Delta S$. Cette éventualité reste à explorer plus en détail.

On examinera ici que l'hypothèse la plus simple ou $\Delta S_{I}$ et $\Delta S_{I H}$ sont des paramètres indépendants.

On trouve alors selon la méthode exposée ci-devant :

$$
\Delta S_{H}^{*}=\left(\sqrt{\frac{T_{H S i}}{T_{0}}} \sqrt{1+\frac{\Delta S_{I H}}{G_{H}}}-1\right) G_{H}
$$

L'entropie de transfert optimale est proportionnelle à $G_{H}$ et augmente avec $\Delta S_{I H}$. 
Le maximum d'énergie associée MaxW vaut :

$$
\operatorname{MaxW}=G_{H}\left[\left(\sqrt{T_{H S i}}-\sqrt{\left(1+\frac{\Delta S_{I H}}{G_{H}}\right)} \sqrt{T_{0}}\right)^{2}-T_{0} \frac{\Delta S_{I}}{G_{H}}\right]
$$

$M a x W$ est donc une fonction croissante de $G_{H}$, mais décroissante des deux ratios d'irréversibilité $\frac{\Delta S_{I H}}{G_{H}}$ et $\frac{\Delta S_{I}}{G_{H}}$.

Le rendement au maximum de puissance s'en déduit. D'autres cas simples pourrait être fournis (près des auteurs). Ils ne sont pas rapportés ici pour de raisons de brièveté. Des prolongements sont en cours de publication [42-44].

\section{Optimisations séquentielles en énergie de moteur endoréversible adiabatique de Curzon-} Ahlborn

\subsection{Rappel de l'optimisation en énergie selon CURZON-AHLBORN}

Cette optimisation, conforme au schéma présenté sur la Figure 4, comporte deux variables intensives $T_{H}, T_{C}$.

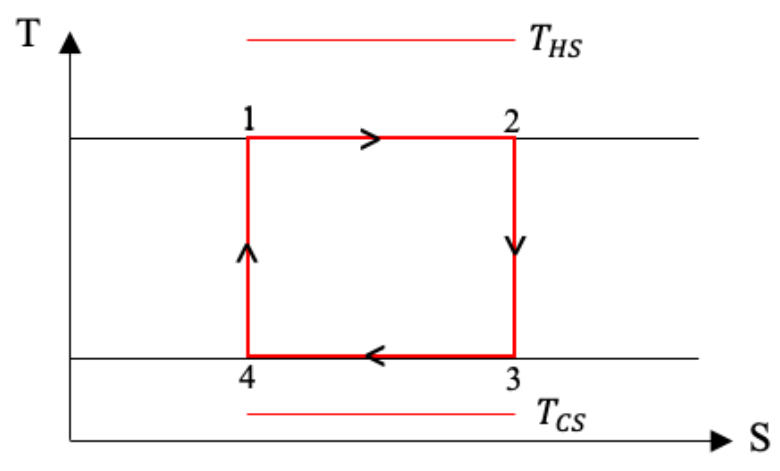

Figure 4 Cycle moteur endoréversible de CARNOT

$T_{H}$, température du fluide cyclé au bout chaud du système, source de chaleur thermostat à $T_{H S}$.

$T_{C}$, température du fluide cyclé au bout froid du système, puits de chaleur thermostat à $T_{C S}$.

En supposant une loi de transfert d'énergie thermique linéaire on a selon [32] :

$$
Q_{i}=G_{i} \Delta T_{i}
$$

avec $G_{i}=K_{i}^{\prime} t_{i}$, conductance de transfert d'énergie thermique $(\mathrm{J} / \mathrm{K})$.

Il vient :

$$
\begin{gathered}
Q_{H}=G_{H}\left(T_{H S}-T_{H}\right) ; G_{H}=K_{H}^{\prime} t_{H} \\
Q_{C}=G_{C}\left(T_{C}-T_{C S}\right) ; G_{C}=K_{C}^{\prime} t_{C}
\end{gathered}
$$

La conservation de l'énergie, d'après la première loi de la Thermodynamique, impose alors pour l'énergie mécanique $W$ : 


$$
W=Q_{H}-Q_{C}
$$

Alors que le second principe de la Thermodynamique conduit au bilan entropique de cycle pour le convertisseur endo-réversible :

$$
\frac{Q_{H}}{T_{H}}=\frac{Q_{C}}{T_{C}}
$$

Il est alors facile de montrer [31] que le maximum d'énergie mécanique, en fonction des variables $T_{H}, T_{C}$ est tel que :

$$
\begin{aligned}
& T_{H}^{*}=\sqrt{T_{H S}} \frac{G_{H} \sqrt{T_{H S}}+G_{C} \sqrt{T_{C S}}}{G_{H}+G_{C}} \\
& T_{C}^{*}=\sqrt{T_{C S}} \frac{G_{H} \sqrt{T_{H S}}+G_{C} \sqrt{T_{C S}}}{G_{H}+G_{C}} \\
& M_{A} X_{1}(W)=\frac{G_{H} G_{C}}{G_{H}+G_{C}}\left(\sqrt{T_{H S}}-\sqrt{T_{C S}}\right)^{2}
\end{aligned}
$$

On peut alors tracer l'évolution de ce premier maximum d'énergie adimensionné en fonction du rapport des températures $\frac{T_{H S}}{T_{C S}}$ (on choisit ici de manière arbitraire les conductances adimensionnées $\left.G_{H_{\text {adim }}}=G_{C_{\text {adim }}}=\frac{1}{2}\right)$ :

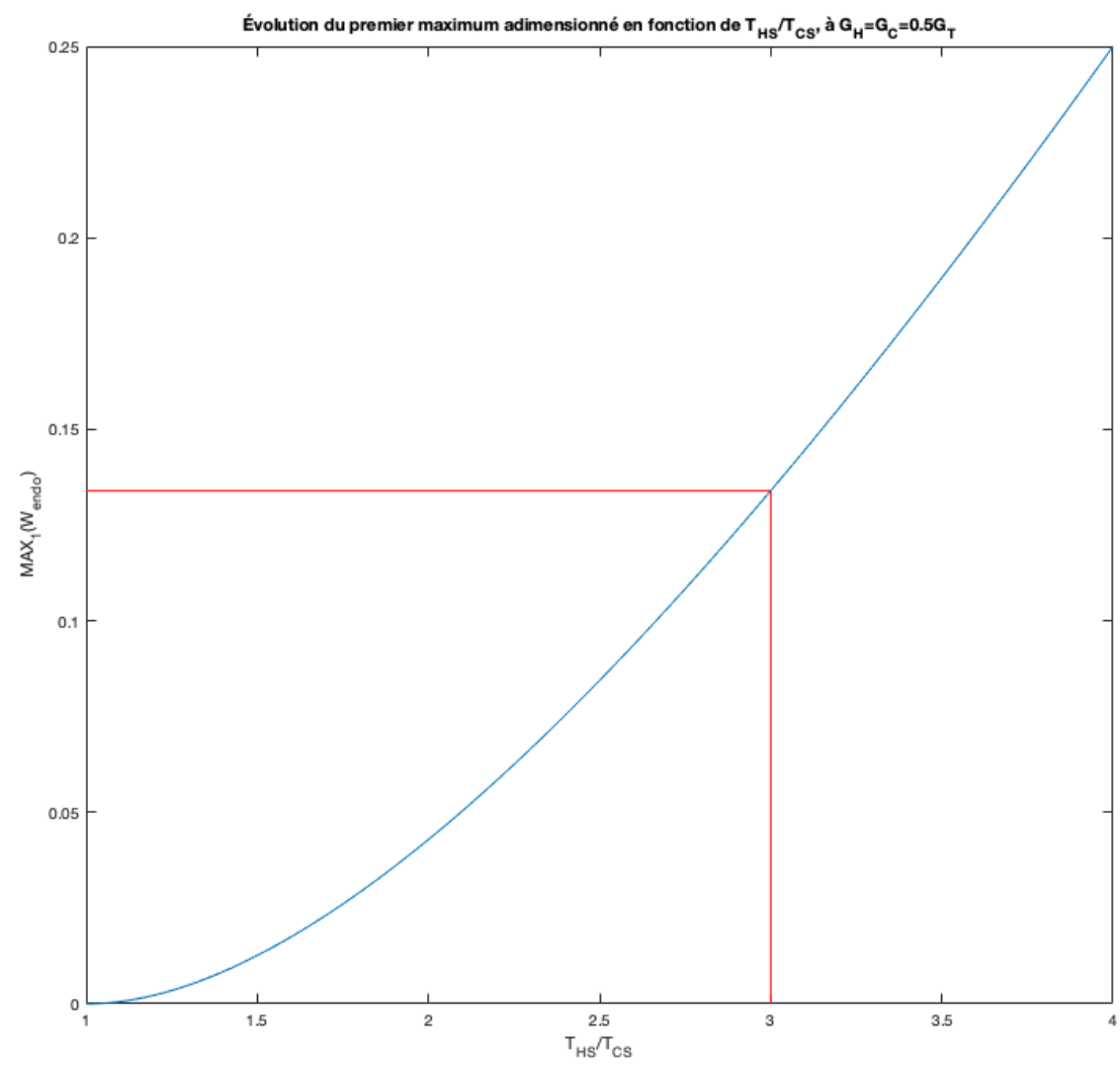

Figure 5. Evolution de $M A X_{1} W$ en fonction du rapport des températures de source et de puits à l'équipartition des conductances

On rappelle que l'ordre de grandeur du rapport $\frac{T_{H S}}{T_{C S}}$ est voisin de 3 pour un moteur à combustion. Cela nous donne une valeur du maximum adimensionné proche de 0,13. Pour obtenir la valeur du maximum énergétique il faut multiplier cette valeur par $T_{C S} G_{H}$, le produit de la température du puits de chaleur thermostat et de la conductance de transfert d'énergie thermique choisie en référence. 
Ce maximum d'énergie est associé au rendement suivant :

$$
\eta_{I}\left(M A X_{1} W\right)=1-\sqrt{\frac{T_{C S}}{T_{H S}}}
$$

On rappelle ici que $G_{H}, G_{C}$ sont les conductances de transfert d'énergie thermique telles qu'énoncées dans les relations [41] et [42]. Ces conductances caractérisent des transformations isothermes et non des valeurs moyennes sur le cycle conformément à :

$$
G_{H}=K_{H} \tau ; G_{C}=K_{C} \tau
$$

avec $\tau$, durée du cycle.

Elles caractérisent donc de façon générale la grandeur associée à une première contrainte de finitude :

$$
G_{H}+G_{C}=G_{T}
$$

\subsection{Première proposition d'optimisation en énergie par rapport aux variables finies $G_{H}, G_{C}$}

Cette optimisation précède celle proposée par Curzon-Ahlborn qui suivra. La fonction objectif étant l'énergie mécanique produite (43), la combinaison de $(41,42,43,44)$ a conduit à (47) nouvelle fonction objectif en séquence dont on cherche le maximum en présence de la contrainte de dimension finie (49).

Il est facile de voir que $M A X_{2}(W)$ se produit à l'équipartition des $G_{i}$ :

$$
G_{H}^{*}=G_{C}^{*}=\frac{G_{T}}{2}
$$

Ce résultat est corroboré par ceux obtenus avec MATLAB, dont on peut voir ci-dessous (Figure 6) une illustration :

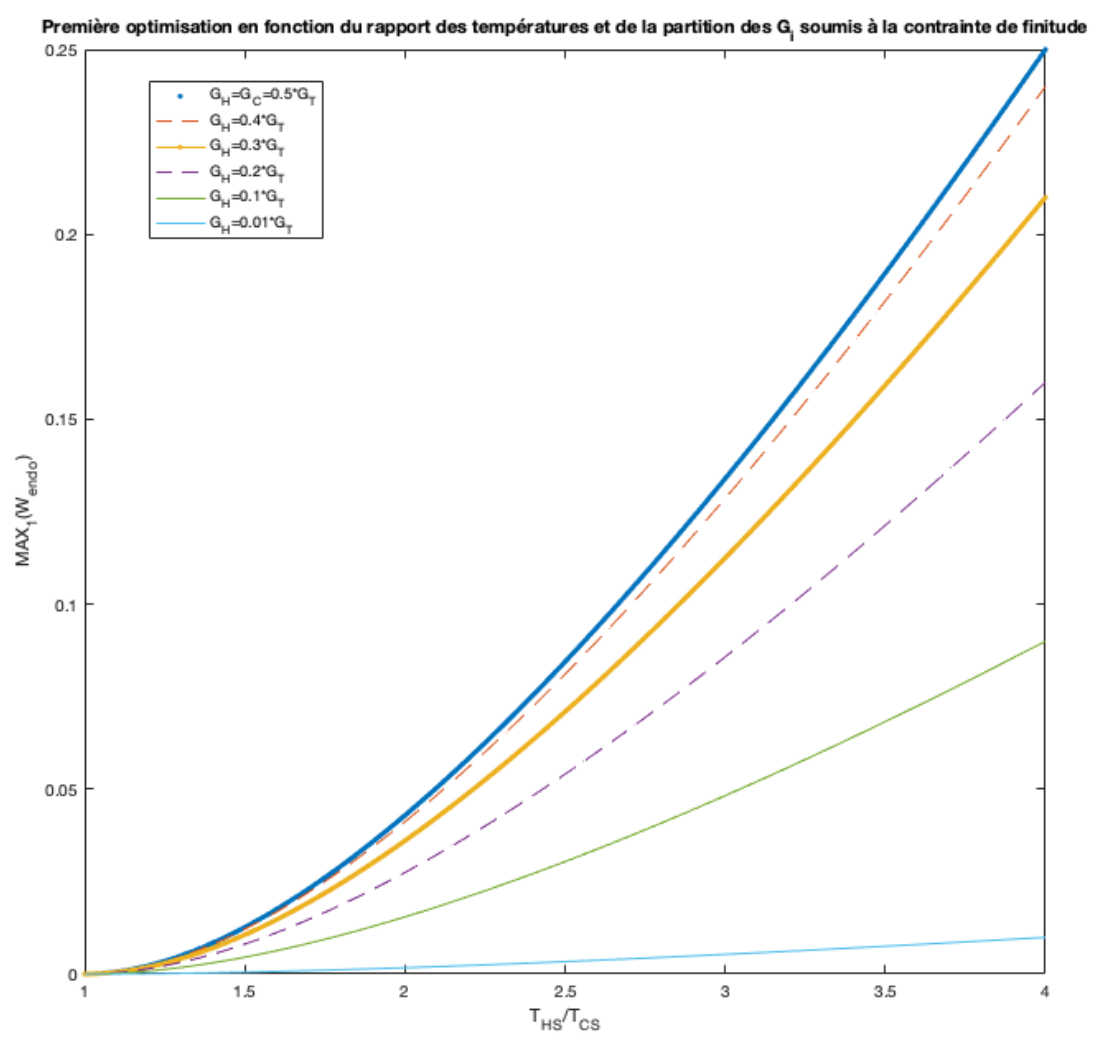

Figure 6. Influence de la partition des conductances sur $M A X_{1} W$ 
La deuxième optimisation en température conduit à :

$$
\begin{aligned}
& T_{H}^{*}=\sqrt{T_{H S}} \frac{\sqrt{T_{H S}}+\sqrt{T_{C S}}}{2} \\
& T_{C}^{*}=\sqrt{T_{C S}} \frac{\sqrt{T_{H S}}+\sqrt{T_{C S}}}{2} \\
& M A X_{2}(W)=\frac{G_{T}}{4}\left(\sqrt{T_{H S}}-\sqrt{T_{C S}}\right)^{2}
\end{aligned}
$$

On a alors :

$$
\eta_{I}\left(M A X_{2}(W)\right)=1-\sqrt{\frac{T_{C S}}{T_{H S}}}
$$

Le résultat de Curzon-Ahlborn avec cette première condition de finitude (49) et la puissance du moteur correspondant à $M A X_{2}(W)$ vaut :

$$
\overline{\dot{W}}=\frac{G_{T}}{4 \tau}\left(\sqrt{T_{H S}}-\sqrt{T_{C S}}\right)^{2}
$$

\subsection{L'optimisation en puissance motrice par rapport aux variables $t_{H}, t_{C}$ en temps fini soumis à la contrainte de finitude (4)}

Contrairement à la démonstration proposée dans [32] qui est une optimisation par rapport aux variables $T_{H}, T_{C}, t_{H}, t_{C}$, nous proposons ici une optimisation séquentielle par rapport aux variables $T_{H}, T_{C}$ qui fournit le maximum d'énergie (47) comme vu ci-devant et par conséquent la puissance moyenne à ce maximum d'énergie :

$$
\overline{\dot{W}}\left(M A X_{1}(W)\right)=\frac{K_{H}^{\prime} K_{C}^{\prime} t_{H} t_{C}}{G_{T} \alpha\left(t_{H}+t_{C}\right)}\left(\sqrt{T_{H S}}-\sqrt{T_{C S}}\right)^{2}
$$

avec :

$$
\begin{aligned}
& G_{T}=K_{H}^{\prime} t_{H}+K_{C}^{\prime} t_{C} \\
& \tau=\alpha\left(t_{H}+t_{C}\right)=\alpha \tau^{\prime}
\end{aligned}
$$

Mais (56) est une contrainte de finitude en $G_{i}$ alors que (4) est une contrainte de finitude en temps ( $\tau$ et $\alpha$ paramètres). Curzon et Ahlborn ne prennent en compte que la contrainte en temps. De ce fait (56) est relaxée et la puissance moyenne devient :

$$
\bar{W}\left(M A X_{1}(W)\right)=\frac{K_{H}^{\prime} K_{C}^{\prime} t_{H} t_{C}}{\left(K_{H}^{\prime} t_{H}+K_{C}^{\prime} t_{C}\right)} \frac{\left(\sqrt{T_{H S}}-\sqrt{T_{C S}}\right)^{2}}{\tau}
$$

On voit donc que le maximum de puissance moyenne de cycle $\overline{\dot{W}}$ correspond au minimum de $\left(\frac{1}{K_{H}^{\prime} t_{H}}+\frac{1}{K_{C}^{\prime} t_{C}}\right)$ soumis à la contrainte (4). La méthode des multiplicateurs de Lagrange permet de trouver que :

$$
\begin{aligned}
& t_{H}^{*}=\frac{\tau^{\prime} \sqrt{K_{C}^{\prime}}}{\sqrt{K_{H}^{\prime}}+\sqrt{K_{C}^{\prime}}} \\
& t_{C}^{*}=\frac{\tau^{\prime} \sqrt{K_{H}^{\prime}}}{\sqrt{K_{H}^{\prime}}+\sqrt{K_{C}^{\prime}}}
\end{aligned}
$$




$$
\operatorname{MAX}\left\{\overline{\dot{W}}\left(M A X_{1}(W)\right)\right\}=\frac{K_{H}^{\prime} K_{C}^{\prime}}{\alpha\left(\sqrt{K_{H}^{\prime}}+\sqrt{K_{C}^{\prime}}\right)^{2}}\left(\sqrt{T_{H S}}-\sqrt{T_{C S}}\right)^{2}
$$

Le résultat est obtenu dans [32]. Mais comme annoncé dans l'introduction, une optimisation par rapport aux grandeurs finies $K^{\prime}{ }_{i}$ munie de la contrainte de finitude de (6) est possible, tant en puissance qu'en énergie. Le $M A X_{2}(W)$ ou $M A X_{2}(\overline{\dot{W}})$ correspond au minimum de $\left(\frac{1}{\sqrt{K_{H}^{\prime}}}+\frac{1}{\sqrt{K_{C}^{\prime}}}\right)$ avec la contrainte (6).

On trouve par le calcul variationnel l'équipartition des conductances d'énergie thermique :

$$
K_{H}^{\prime}=K_{C}^{\prime}=\frac{K_{T}}{2}
$$

Ce résultat est corroboré par ceux obtenus avec MATLAB, dont on peut voir ci-dessous (Figure 7) l'illustration graphique :

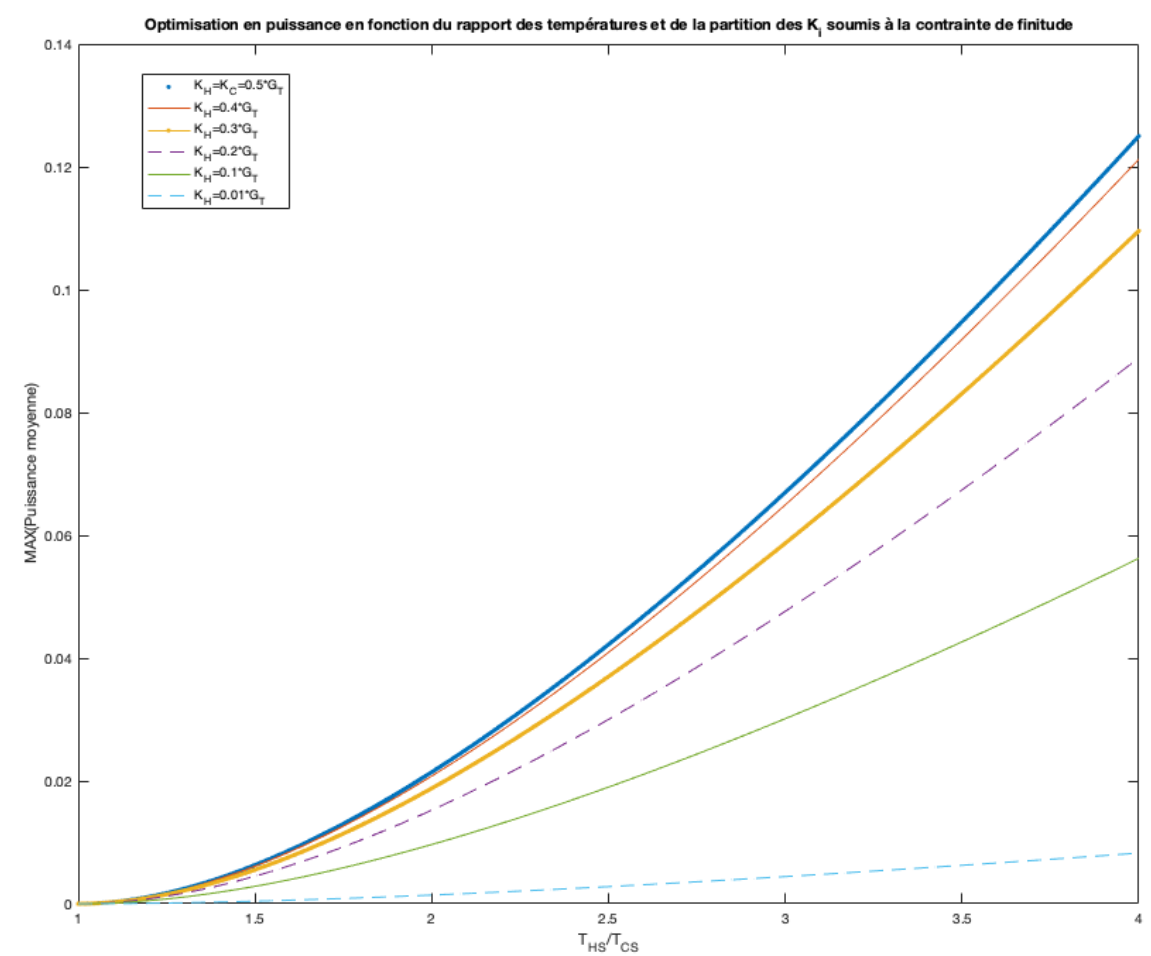

Figure 7. Influence de la partition des conductances sur $M A X_{2}(W)$

$$
M A X_{3}(W)=\frac{K_{T} \tau^{\prime}}{8}\left(\sqrt{T_{H S}}-\sqrt{T_{C S}}\right)^{2}
$$

Le rendement de ce troisième maximum est inchangé :

$$
\begin{aligned}
& \eta_{I}\left(M A X_{3}(W)\right)=1-\sqrt{\frac{T_{C S}}{T_{H S}}} \\
& \operatorname{MAX}_{2}(\overline{\dot{W}})=\frac{K_{T}^{\prime}}{8 \alpha}\left(\sqrt{T_{H S}}-\sqrt{T_{C S}}\right)^{2}
\end{aligned}
$$

Le résultat est vrai quelque soit la séquence. 


\section{Optimisation séquentielle en énergie et puissance du moteur de Curzon-Ahlborn modifié}

On reprend ici la même méthodologie qu'au paragraphe 4.2, mais en considérant des moyennes de cycle comme proposées en (48).

L'optimisation par rapport aux température $T_{H}, T_{C}$ variables ne change pas et fournit le premier maximum en énergie (47). La combinaison de (48) et (49) conduit à :

$$
M A X_{1}(W)=\frac{K_{H} K_{C} \tau}{K_{H}+K_{C}}\left(\sqrt{T_{H S}}-\sqrt{T_{C S}}\right)^{2}
$$

On rappelle que le rendement associé est :

$$
\eta_{I}(M A X W)=1-\sqrt{\frac{T_{C S}}{T_{H S}}}
$$

On voit alors sur l'expression (64) que le maximum d'énergie du moteur endoréversible coincide avec le maximum de puissance.

En revanche l'optimisation successive porte ici sur les variables en valeur moyenne sur le cycle $\left(K_{H}\right.$, $\left.K_{C}\right)$ ou $\left(A_{H}, A_{C}\right)$ sachant que classiquement on a :

$$
\begin{aligned}
& K_{H}=k_{H} A_{H} \\
& K_{C}=k_{C} A_{C}
\end{aligned}
$$

$A_{H}, A_{C}$ sufaces respectives des échangeurs chaud et froid ;

$k_{H}, k_{C}$ coefficients classiques de transfert de chaleur global aux bouts chaud et froid.

$$
M A X_{1}\left(W_{\text {endo }}\right)=\frac{k_{H} k_{C} A_{H} A_{C}\left(\sqrt{T_{H S}}-\sqrt{T_{C S}}\right)^{2} \tau}{k_{H} A_{H}+k_{C} A_{C}}
$$

La méthode des multiplicateurs de Lagrange fournit la distribution optimale des surfaces de transfert $A_{H}^{*}, A_{C}^{*}$ :

$$
\begin{aligned}
& A_{H}^{*}=A_{T} \frac{\sqrt{k_{C}}}{\sqrt{k_{H}}+\sqrt{k_{C}}} \\
& A_{C}^{*}=A_{T} \frac{\sqrt{k_{H}}}{\sqrt{k_{H}}+\sqrt{k_{C}}}
\end{aligned}
$$

Avec :

$$
\operatorname{MAX}_{2}\left(W_{\text {endo }}\right)=\frac{k_{H} k_{C} A_{T} \tau\left(\sqrt{T_{H S}}-\sqrt{T_{C S}}\right)^{2}}{\left(\sqrt{k_{H}}+\sqrt{k_{C}}\right)^{2}}
$$

À cette deuxième optimisation en énergie correspond le rendement endoréversible de CARNOT :

$$
\eta_{I}\left(M_{2}\left(W_{\text {endo }}\right)\right)=1-\sqrt{\frac{T_{C S}}{T_{H S}}}
$$

On peut alors faire varier le rapport $\frac{k_{H}}{k_{C}}$ pour voir comment évolue $M A X_{2}\left(W_{\text {endo }}\right)$. Cela est représenté sur la Figure 8. 


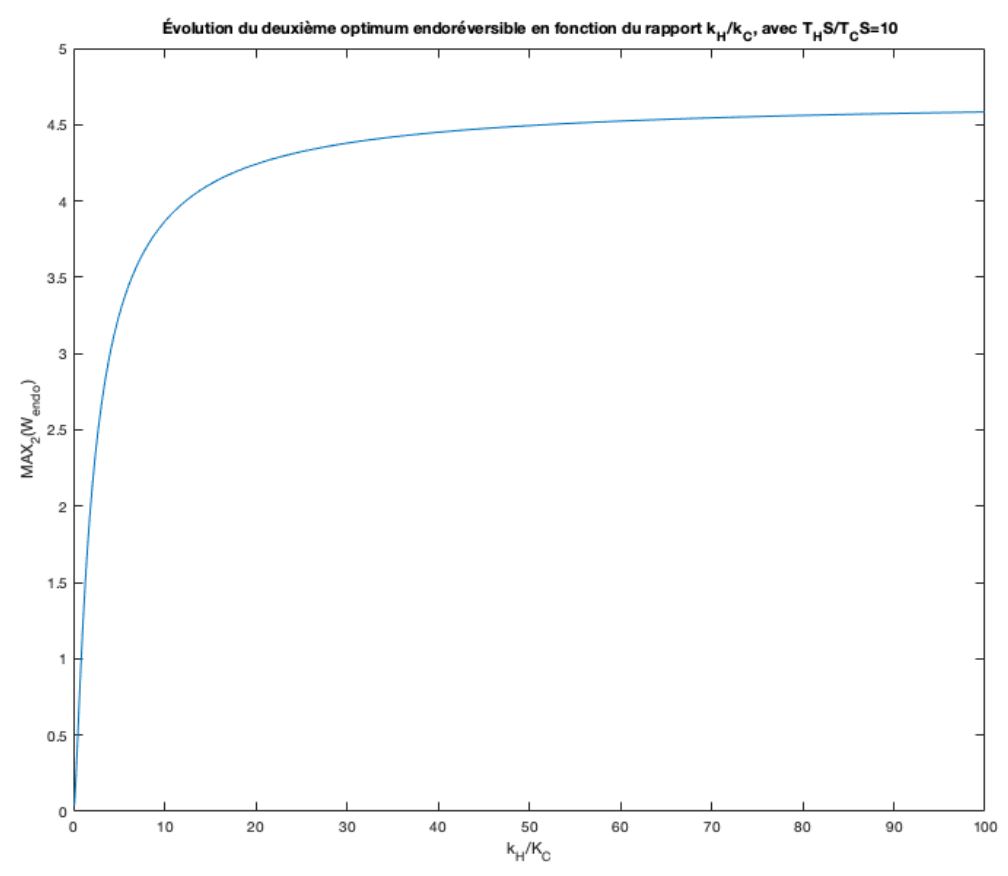

Figure 8. Evolution de $\mathrm{MAX}_{2}\left(W_{\text {endo }}\right)$ en fonction du rapport des coefficients d'échange de chaleur

La valeur adimensionnée de $M A X_{2}\left(W_{\text {endo }}\right)$ tend vers une limite en $+\infty$, proche de 4,6 . On observe une cassure sur la représentation graphique: en effet le coefficient directeur de la courbe de $M A X_{2}\left(W_{\text {endo }}\right)$ est intéressant jusqu'au rapport $\frac{k_{H}}{k_{C}}=20$. Au-delà le coût de transfert chaud devient trop important pour le gain energétique que l'on peut espérer. En effet il n'existe pas de source d'énergie infinie dans la nature, à fortiori dans l'industrie. Le raisonnement que nous adopterons fait apparaître une contrainte de finitude qui traduit ce fait.

Une dernière optimisation est possible par réglage des coefficients de transfert de chaleur globaux, si ceux-ci sont soumis à une contrainte de finitude :

$$
k_{H}+k_{C}=k_{T}
$$

En effet $\operatorname{MAX}_{3}\left(W_{\text {endo }}\right)$ correspond à $\min \left(\frac{\sqrt{k_{H}}+\sqrt{k_{C}}}{\sqrt{k_{H}} \sqrt{k_{C}}}\right)=\min \left(\frac{1}{\sqrt{k_{H}}}+\frac{1}{\sqrt{k_{C}}}\right)$. Voir l'annexe pour l'optimisation en $k_{H}, k_{C}$.

\section{Discussion - conclusion}

Le présent article propose une reconsidération complète de la proposition de Curzon-Ahlborn qui appartient à la Thermodynamique en Temps Fini (T.T.F.) endoréversible.

Cette reconsidération est resituée par rapport à un ensemble de references conséquent (50 articles) montrant l'importance particulière des moteurs thermomécaniques. Mais la publication vise des aspects plus fondamentaux liés à l'energie, l'efficacité (rendement au sens du premier principe) et puissance.Le but était de proposer des critères de qualité du moteur plus proche de la réalité fonctionnelle de celui-ci , par opposition à la limite inateignable du rendement de Carnot et de surcroit a puissance nulle.

En repartant des bases historique et du modèle de moteur de Carnot sans pertes thermmiques, on a montré que pour un moteur endo-irreversible, le rendement energètique fait apparaitre naturellement un ratio entropique précis, comme suggeré par Novikov puis Ibrahim. 
La seconde étape a été de completer le modèle des irreversibilités de convertissuer par l'irreversibilité de transfert thermique à la source ( moteur de Chambadal amélioré). Il apparait alors de facon générique une conductance généraliséee de transfert thermique $\mathrm{G}$, valable quelque soit la loi de transfert. Cette approche donne lieu à diverses optimisations séquentielles qui sont dépendantes des contraintes de finitude.

De plus nous avons montré que le couplage des transferts thermiques entre la source (le donneur thermique) et le fluide cyclé ( récepteur thermique) ajoute une contrainte permettant de passer d'une variable de température à une variable entropique dont le choix de référence est multiple et donne encore lieu à développement. Une illustration en a été donnée au paragraphe 3.2.1.

La reprise des modèles de Carnot, Chambadal, Curzon-Ahlborn nous a amené à préciser la différence entre moyenne de transformation et moyenne de cycle: cette différence est en effet essentielle.

En conservant l'approche de Curzon-Ahlborn, nous avons montré qu'il était possible d'ajouter une optimisation séquentielle complémentaire par rapport aux variables conductances de transfert thermique des transformations isothermes, lorsqu'on caractérise la puissance moyenne. La distribution optimale des durées et des conductances conduit alors à la puissance maximale (paragraphe 4) :

$$
\operatorname{MAX}_{2}(\overline{\dot{W}})=\frac{K_{T}^{\prime}}{8 \alpha}\left(\sqrt{T_{H S}}-\sqrt{T_{C S}}\right)^{2}
$$

Si on raisonne sur les moyennes de cycle, on a vu que rechercher le maximum d'énergie coïncide avec la recherche du maximum de puissance moyenne. Il existe une distribution optimale des surfaces de transfert (paragraphe 5).

En résumé , la reprise du modèles de Curzon-Ahlborn a permis de preciser la différence entre moyenne de cycle ( aproche de Chambadal que nous preconisons) et moyenne de transformation (approche de Curzon-Ahlborn).

Ces résultats forment un ensemble plus détaillé et plus complet que les résultats primitifs de CurzonAhlborn. Nos études présentes visent à les prolonger encore et sont en cours d'extension et de publication.

\section{Annexe A}

Optimisation en $k_{H}, k_{C}$ pour le cas endoreversible :

$$
\begin{aligned}
& L\left(k_{H}, k_{C}\right)=\left(\frac{1}{\sqrt{k_{H}}}+\frac{1}{\sqrt{k_{C}}}\right)+\lambda\left(k_{H}+k_{C}-k_{T}\right) \\
& \frac{\partial L}{\partial k_{H}}=-\frac{1}{2} k_{H}^{-\frac{3}{2}}+\lambda \\
& \frac{\partial L}{\partial k_{C}}=-\frac{1}{2} k_{C}^{-\frac{3}{2}}+\lambda
\end{aligned}
$$

Donc :

$$
k_{H}^{*}=k_{C}^{*}=\frac{k_{T}}{2}
$$

ce qui correspond à l'équipartition des coefficients de transfert thermique globaux en endo-réversible.

Il vient alors : 


$$
\begin{aligned}
& M A X_{3}\left(W_{\text {endo }}\right)=\frac{k_{T}^{2} A_{T} \tau\left(\sqrt{T_{H S}}-\sqrt{T_{C S}}\right)^{2}}{4\left(\sqrt{\frac{k_{T}}{2}}+\sqrt{\frac{k_{T}}{2}}\right)^{2}} \\
& M^{2} X_{3}\left(W_{\text {endo }}\right)=\frac{k_{T} A_{T} \tau\left(\sqrt{T_{H S}}-\sqrt{T_{C S}}\right)^{2}}{8}
\end{aligned}
$$

Ce résultat vaut aussi pour l'optimum en puissance.

Le rendement associé vaut toujours le rendement de Carnot endoréversible :

$$
\eta_{I}\left(M A X_{3}\left(W_{\text {endo }}\right)\right)=1-\sqrt{\frac{T_{C S}}{T_{H S}}}
$$

\section{Bibliographie}

[1] M. Feidt, Optimisation d'un cycle de Brayton moteur en contact avec des capacités thermiques finies, Rev. Gen. Therm. 35 (1996) 662-666. https://doi.org/10.1016/S0035-3159(96)80063-8.

[2] C-K. Chen, Y-F. Su, Exergetic efficiency optimization for an irreversible Brayton refrigeration cycle, Int. J. Therm. Sci. 44 (2005) 303-310. https://doi.org/10.1016/j.ijthermalsci.2004.09.003.

[3] W. Wang, L. Chen, F. Sun, C. Wu, Power optimization of an endoreversible closed intercooled regenerated Brayton cycle, Int. J. Therm. Sci. 44 (2005) 89-94. https://doi.org/10.1016/j.ijthermalsci.2004.06.002.

[4] Y. Ust, B. Sahin, A. Kodal, Performance analysis of an irreversible Brayton heat engine based on ecological coefficient of performance criterion, Int. J. Therm. Sci. 45 (2006) 94-101. https://doi.org/10.1016/j.ijthermalsci.2005.04.005.

[5] S.K. Tyagi, G.M. Chen, Q. Wang, S.C. Kaushik, Thermodynamic analysis and parametric study of an irreversible regenerative-intercooled-reheat Brayton cycle, Int. J. Therm. Sci. 45 (2006) 829-840. https://doi.org/10.1016/j.ijthermalsci.2005.10.011.

[6] P. Remy, Y. Boissenin, M. Molière, Gas turbine and cogeneration installations of Complex Construction - The UEM Power-Plant case, Rev. Gen. Therm. 33 (1994) 717-725.

[7] M. Moliere, Stationary gas turbines and primary energies: A review of fuel influence on energy and combustion performances, Int. J. Therm. Sci. 39 (2000) 141-172. https://doi.org/10.1016/S1290-0729(00)00236-2.

[8] S. Xia, L. Chen, F. Sun, Engine performance improved by controlling piston motion: linear phenomenological law system Diesel engine, Int. J. Therm. Sci. 51 (2012) 163-174. https://doi.org/10.1016/j.ijthermalsci.2011.08.005.

[9] U. Asad, M. Zheng, Fast heat release characterization of a diesel engine, Int. J. Therm. Sci. 47 (2008) 1688-1700. https://doi.org/10.1016/j.ijthermalsci.2008.01.009.

[10] F.E. Czarneski, S.H. Och, L.M. Moura, E. Domingues, Experimental model for prediction of tip temperature of diesel injector nozzle in dual-fuel engines, Int. J. Therm. Sci. 138 (2019) 596-604. https://doi.org/10.1016/j.ijthermalsci.2018.12.034.

[11] P. Chesse, J.F. Hetet, X. Tauzia, J.P. Frayret, Influence of the engine/compressor duct on the operating range of a high output Diesel engine with a two-stage turbo charging system, Rev. Gen. Therm. 37 (1998) 801-812. https://doi.org/10.1016/S0035-3159(98)80006-8.

[12] Y. Zhou, S.K. Tyagi, J. Chen, Performance analysis and optimum criteria of an irreversible Braysson heat engine, Int. J. Therm. Sci. 43 (2004) 1101-1106. https://doi.org/10.1016/j.ijthermalsci.2004.02.008.

[13] J.C. Lin, S.S. Hou, Performance analysis of an air-standard Miller cycle with considerations of heat loss as a percentage of fuel's energy, friction and variable specific heats of working fluid, Int. J. Therm. Sci. 47 (2008) 182-191. https://doi.org/10.1016/j.ijthermalsci.2007.02.002.

[14] Y. Zhao, J. Chen, An irreversible heat engine model including three typical thermodynamic cycles and their optimum performance analysis, Int. J. Therm. Sci. 46 (2007) 605-613. https://doi.org/10.1016/j.ijthermalsci.2006.04.005.

[15] G. Popescu, V. Radcenco, M. Costea, M. Feidt, Optimisation thermodynamique en temps finie du moteur de Stirling endo- et exo-réversible, Rev. Gen. Therm. 35 (1996) 656-661. https://doi.org/10.1016/S00353159(96)80062-6.

[16] S. Loizeau, Thermodynamic approach to the fuel-cell considered as an open system, Rev. Gen. Therm. 34 (1995) 426-436. 
[17] V. Radcenco, E.E. Vasilescu, G. Popescu, V. Apostol, New approach to thermal power plants operation regimes maximum power versus maximum efficiency, Int. J. Therm. Sci. 46 (2007) 1259-1266. https://doi.org/10.1016/j.ijthermalsci.2007.01.022.

[18] H.H. Erdem, A.V. Akkaya, B. Cetin, A. Dagdas, S.H. Sevilgen, B. Sahin, I. Teke, C. Gungor, S. Atas, Comparative energetic and exergetic performance analysis for coal-fired thermal power plants in Turkey, Int. J. Therm. Sci. 48 (2009) 2179-2186. https://doi.org/10.1016/j.ijthermalsci.2009.03.007.

[19] W.D. Wu, H. Zhang, C.L. Men, Performance of a modified zeolite 13X-water adsorptive cooling module powered by exhaust waste heat, Int. J. Therm. Sci. 50 (2011) 2042-2049. https://doi.org/10.1016/j.ijthermalsci.2011.05.005.

[20] M. Barrère, Le rôle du temps dans l'optimisation des cycles thermodynamiques, Rev. Gen. Therm. Fr. 228 (1980) 995-1006.

[21] M. Barrère, La thermoéconomie, Rev. Gen. Therm. Fr. 225 (1983) 243-262.

[22] W. Nie, J. He, X. Deng, Local stability of an irreversible Carnot heat engine, Int. J. Therm. Sci. 47 (2008) $663-$ 640. https://doi.org/10.1016/j.ijthermalsci.2007.04.004.

[23] E. Fratzscher, Exergy and possible applications, Rev. Gen. Therm. 36 (1997) 690-696. https://doi.org/10.1016/S0035-3159(97)88433-4.

[24] G. Grazzini, F. Gori, Influence of thermal irreversibilities on work producing systems, Rev. Gen. Therm. 312 (1987) 637-639.

[25] U. Lucia, G. Grazzini, Global analysis of dissipation due to irreversibility, Rev. Gen. Therm. 36 (1997) 605-609. https://doi.org/10.1016/S0035-3159(97)89987-4.

[26] P. Goffause, How to finance the energy future of greater Europe and respect the environment, Rev. Gen. Therm. 33 (1994) 214-216.

[27] M. Roche, Récupération de chaleur, le cycle thermodynamique idéal, Rev. Gen. Therm. Fr. 260-261 (1983) 561571.

[28] D. Stitou, M. Feidt, Nouveaux critères pour l'optimisation et la caractérisation des procédés thermiques de conversion énergétique, Int. J. Therm. Sci. $44 \quad$ (2005) 1142-1153. https://doi.org/10.1016/j.ijthermalsci.2005.08.013.

[29] M. Barrère, Démarche unitaire de la Thermodynamique dans l'étude des systèmes complexes, Rech. Aerospatiale, 5 (1995) 353-362.

[30] F. Arias, On osmotic heat engines driven by thermal precipitation-dissolution of saturated aqueous solution, Int. J. Therm. Sci. 133 (2018) 151-161. https://doi.org/10.1016/j.ijthermalsci.2018.07.024.

[31] M. Feidt, Thermodynamique et optimisation énergétique des systèmes et procédés, $1^{\text {er }}$ éd., Lavoisier TEC et DOC, Paris, 1987 (et éditions suivantes 1996, 2016).

[32] F.L. Curzon, B. Ahlborn, Efficiency of a Carnot engine at maximum power output, Am. J. Phys. 43 (1975) 22-24. https://doi.org/10.1119/1.10023.

[33] B. Andresen, Finite-Time Thermodynamics, Physics Laboratory II, University of Copenhagen, 1983.

[34] P. Chambadal, Les centrales nucléaires, Armand Colin, Paris, 1957, 41-58.

[35] I. Novikov, The efficiency of atomic power stations, At. Energy 3 (1957) 409-412 https://doi.org/10.1007/BF01507240.

[36] A. Vaudrey, F. Lanzetta, M. Feidt, H. B. Reitlinger and the origin of the efficiency at maximum power for heat engines, J. of Non-Equil. Thermodyn. 39 (2014) 199-203. https://doi.org/10.1515/jnet-2014-0018.

[37] J. Moutier, Eléments de Thermodynamique, Gauthier-Villars, Paris, 1872.

[38] L. Ser, Traité de physique industrielle : production et utilisation de la chaleur (Machines à air chaud), G. Masson, Paris, 1888, 842-859.

[39] O.M. Ibrahim, S. Klein, J.W. Mitchell, Optimum heat power cycles for specified boundary conditions, J. Eng. Gas Turb. Power, 113 (1991) 514-521, https://doi.org/10.1115/1.2906271.

[40] M. Feidt, Thermodynamique optimale en dimensions physiques finies, Hermes, Paris, 2013.

[41] S. Petrescu, M. Costea, M. Feidt, I. Ganea, N. Boriaru, Advanced Thermodynamics of irreversible processes with finite speed and finite dimensions, AGIR, Bucarest, 2015.

[42] M. Feidt, M. Costea, A new perspective on Carnot and Curzon-Ahlborn models of thermomechanical engine by transfer and production of entropy, 15th Joint European Thermodynamic Conference, JETC 2019, May 22-24, 2019, Barcelona, Spain.

[43] M. Feidt, M. Costea, Progress in Carnot and Chambadal modeling of thermomechanical engine by considering entropy production and heat transfer entropy, Entropy 21 (2019) 1232. https://doi.org/10.3390/e21121232.

[44] M. Feidt, R. Feidt, Du rôle fondamental de l'entropie de transfert de chaleur et de la production d'entropie dans la description et l'optimisation des moteurs thermodynamiques : le cas emblématique de la machine de Carnot, C. R. Acad. Sci., in press. 
[45] M. Feidt, Thermodynamics of energy systems and processes: a review and perspectives, J. Appl. Fluid Mech., 5 (2012) 85-98, https://doi.org/.10.36884/jafm.5.02.12171.

[46] M. Blaise, Contribution au développement des moteurs à apport de chaleur externe utilisés pour la conversion des rejets thermiques industriels en électricité. Modélisation et optimisation thermodynamique d'un nouveau convertisseur : Turbosol, Thèse de doctorat, Université de Lorraine, 2016.

[47] M. Feidt, M. Costea, C. Stanciu, D. Stanciu, Entropic model and optimization of a refrigeration machine, IOP Conference Series: Materials Science and Engineering, 595 (2019), https://doi.org/10.1088/1757$\underline{899 X / 595 / 1 / 012025 .}$.

[48] M. Feidt, M. Costea, S. Petrescu, C. Stanciu, Nonlinear Thermodynamic Analysis and Optimization of a Carnot Engine Cycle, Entropy, 18 (2016) 243, https://doi.org/10.3390/e18070243.

[49] M. Feidt, Reconsideration of efficiency of processes and systems from a non-equilibrium point of view, Int. J. Energy Environ. Econ., 11 (2001) 31-49.

[50] M. Feidt, M. Costea, From Finite Time to Finite Physical Dimensions Thermodynamics: The Carnot Engine and Onsager's Relations Revisited, J. Non-Equil. Thermodyn., 43 (2018) 151-161, https://doi.org/10.1515/jnet-20170047. 